\title{
Quantitative analysis of the molding of nanostructures
}

\author{
H. Schift, ${ }^{\text {a) }}$ C. David, and J. Gobrecht \\ Laboratory for Micro- and Nanotechnology, Paul Scherrer Institute, CH-5232 Villigen-PSI, Switzerland
}

\author{
A. D' Amore, D. Simoneta, and W. Kaiser \\ Kunststoff-Ausbildungs-und Technologiezentrum KATZ, CH-5000 Aarau, Switzerland \\ M. Gabriel \\ AWM Werkzeugbau, CH-5630 Muri AG, Switzerland
}

(Received 1 June 2000; accepted 8 September 2000)

\begin{abstract}
Mass fabrication processes such as injection molding and hot embossing are highly suitable for the replication of micro- and nanostructures. They require thermoplastic polymers with good molding properties at moderate process temperatures. We show a quantitative approach to the analysis of the molding properties of polymers in the nanorange in which the parameters are most important for achieving good replication fidelity. With this information we were able to fabricate high precision calibration chips for scanning probe microscopes. (C) 2000 American Vacuum Society.
\end{abstract}

[S0734-211X(00)16506-0]

\section{INTRODUCTION}

Polymer molding is a key technology for the fabrication of devices with nanostructured surfaces. It combines a very high resolution capability with low-cost, mass production potential. It is well established in the microregime, e.g., in compact disk $(\mathrm{CD})$ molding and with current digital versatile disk (DVD) technology, structures with a lateral dimension of $400 \mathrm{~nm}$ and a depth of $100 \mathrm{~nm}$ are now replicated. The fact that the resolution of polymer structures can be below 10 $\mathrm{nm}$ has been know for a long time, ${ }^{1}$ but it has only recently been shown that thermoplastics can be structured to the $\mathrm{nm}$ scale using hot embossing. ${ }^{2,3,4}$ The good molding fidelity of hot embossing has now also been achieved with thermal injection molding, ${ }^{5,6}$ a process which is very demanding in terms of equipment, material and process control.

In the production of CDs, the original structure (the socalled master) is fabricated by laser lithography and electroforming. The microrelief of the original structure is then replicated in an injection molding process and afterwards the processing of the plastic disks is completed with different coating techniques. The grooves which are replicated have aspect ratios (depth-to-width ratio) far below 1 and the inclined walls of the grooves are suitable for good demolding from the master. In order to achieve a high master quality for nanomolding, a direct stamper technique was developed and as shown in Ref. 6 processed silicon wafers were directly used in the molding tool. Apart from quick prototyping using standard cleanroom process equipment, the method has the advantage that high precision nanostructures with aspect ratios higher than 1 can be generated in an easy, straightforward way. Previous work has shown that the replication limit has not yet been reached and that the molding process can be very fast. Initial applications of nanostructured elements, such as calibration structures for scanning probe microscopy (SPM), demonstrated the potential of our process as a fabrication technology. However for future high precision appli-

${ }^{a)}$ Electronic mail: helmut.schift@psi.ch cations it is necessary to broaden our understanding of how nanostructures are molded and which kind of polymers are best suited for nanostructuring. In this article we therefore show how quantitative analysis can help to improve the molding quality of nanostructures.

\section{MOLDING OF NANOSTRUCTURES}

In the past it has been shown which process and material parameters must be controlled for optimal molding. In hot embossing of nanostructures, good molding results can be achieved with a variety of materials and embossing parameters. The key factor for a good and fast molding process is the viscosity of the material, which is largely dependent on the embossing temperature. ${ }^{7}$

As in case of nanoembossing, the replication fidelity using injection molding can be largely controlled by adjusting the mold temperature. In contrast to embossing, where a structure is generated by pressing a structured hot master onto a thermoplastic material, the polymer melt is injected into a large structured mold cavity which has a temperature below the glass transition temperature $\left(T_{g}\right)$ and gives cycle times often in the range of a few seconds. Here, the temperature has to be high enough to ensure a viscous flow of the melt into the nanocavities before it freezes. Therefore incomplete filling of mold cavities can be expected for structures with higher aspect ratios. Nevertheless, with mold temperatures of $125^{\circ} \mathrm{C}$, well under the $T_{g}$ of polycarbonate $\left(148^{\circ} \mathrm{C}\right)$, and an injection pressure of up to $500 \mathrm{bar}$ it was possible to entirely fill nanostructures down to $25 \mathrm{~nm}$ in width, with 40 $\mathrm{nm}$ in depth and with vertical sidewalls. ${ }^{6}$ As long as structures with moderate aspect ratios have to be replicated, injection molding is the method of choice, and it is particularly suitable for large volume fabrication of devices. This is the case for optical structures like those in CD media and diffractive optical elements (DOEs). ${ }^{8}$

In the hot embossing experiments of Ref. 7, the limitation of fabricating structures with higher aspect ratios was mainly attributed to distortion or abrasion of single structures. This 


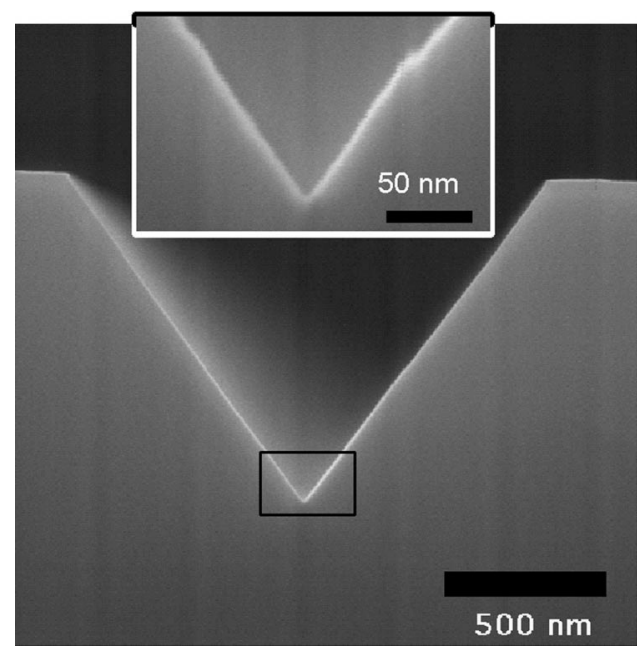

FIG. 1. SEM micrograph of a silicon master stamp featuring a V groove with a sharp bottom. The estimated radius of the silicon tip is $5 \mathrm{~nm}$.

damaging of the molded structure mostly occurs during demolding, and is a result of different effects such as adhesion at the surface, friction due to surface roughness and trapping of the polymer due to negative slopes of cavity sidewalls. Air trapping and contamination of the mold due to clogging may also lead to incomplete molding. In injection molding, the molding process itself is still the main limitation for high aspect ratio structuring, and therefore both the quality of the molding process and the structure geometry have to be improved. Direct control of the replication fidelity of high aspect ratio structures, e.g., by comparing the degree of filling of the mold cavities, is difficult if not impossible without breaking the master or the substrate, and often not reproducible because of structure variations or limitations of the measurement method. Therefore, in the present work lower aspect ratio structures with special geometries were chosen for better demolding and easier analysis.

\section{V-GROOVE STRUCTURES}

Our aim was to generate microstructures on a mold with variable size and defined shape, and to examine a physical property in the nanorange which can be easily measured on the molded structure. Particularly suitable as master structures for this are $\mathrm{V}$ grooves, which can be fabricated in monocrystalline silicon substrates by anisotropical chemical wet etching (see Fig. 1). This process yields grooves with very smooth walls and a sharp V-shaped bottom edge. ${ }^{9}$ Both the volume and the form of the cavity can be precisely determined. Furthermore the molded structures with inclined sidewalls look very similar to the pits of current CDs.

Molded ridges made from $\mathrm{V}$-groove masters are particularly accessible to quantitative atomic force microscopy (AFM) measurements, because the error due to superposition (mathematical convolution) of the shape of the AFM tip with the shape of the ridge is minimized. For nanostructures with a rectangular shape, convolution results in broadening of the structures. For narrow structures the tip may not even reach the bottom of a valley between two ridges in close proximity.

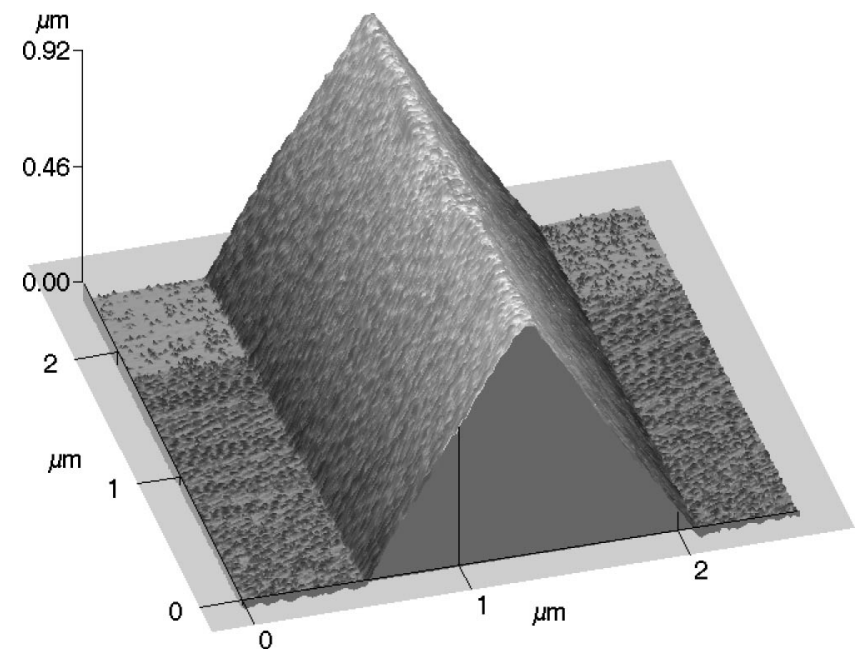

FIG. 2. AFM micrograph of a molded $\Delta$ ridge in PMMA $\left(M_{w} 75 \mathrm{~kg} / \mathrm{mol}\right)$ fabricated in a hot embossing process. The resulting radius of the ridge is $10-20 \mathrm{~nm}$

In Ref. 10, V grooves with a tip radius of less than $10 \mathrm{~nm}$ were replicated using hot embossing (see Fig. 2). The measured radius of the ridge of 10-20 nm lies in the range of the AFM tip radius itself (Park Scientific Instruments Ultralevers $\left.^{\mathrm{TM}}\right)$. It is likely that the actual radius of the ridge is smaller, and that single polymer chain clusters are structured by the molding.

\section{FABRICATION OF NANOSTRUCTURED COMPACT DISKS}

Silicon wafers of $100 \mathrm{~mm}$ in diameter and of $\langle 100\rangle$ orientation were oxidized in a standard process to an oxide thickness of $120 \mathrm{~nm}$. Gratings with periodicities ranging from 120 $\mathrm{nm}$ to $5 \mu \mathrm{m}$ were exposed in resist using e-beam and optical lithography. After development the pattern was transferred into the $\mathrm{SiO}_{2}$ by reactive ion etching using a $\mathrm{CHF}_{3}$ process with a Oxford Plasmalab 100 etcher. The $\mathrm{V}$ grooves were etched in a $20 \% \mathrm{KOH}$ etching solution at $40^{\circ} \mathrm{C}$ and the oxide was then removed in buffered $\mathrm{HF}$ solution resulting in a $\mathrm{V}$-groove structure, shown in Fig. 1. A special tool was developed by AWM Werkzeugbau AG, Muri, Switzerland, which is able to accept structured $100 \mathrm{~mm}$ wafers as inserts. The silicon wafer was held both mechanically and with a vacuum. Injection molding tests were carried out on a Netstal injection molding machine Discjet 600. Polycarbonates Makrolon CD 2005 and DP 1-1265 from Bayer were used as the molding material. ${ }^{11}$ Makrolon DP 1-1265 was developed for DVD molding and exhibits better flow properties. Improved polymer flow is particularly important for the molding of nanostructures and therefore most of the experiments were performed with DP 1-1265. An antiadhesive layer (a fluorinated silane) deposited on the master prior to molding reduced sticking of the disk to the wafer, especially when higher mold temperatures with mold temperatures (up to $130{ }^{\circ} \mathrm{C}$ ) were chosen. The injection molding parameters, injection pressure and speed, switching point and dwell pressure, were adapted to get optimal filling and demolding con- 


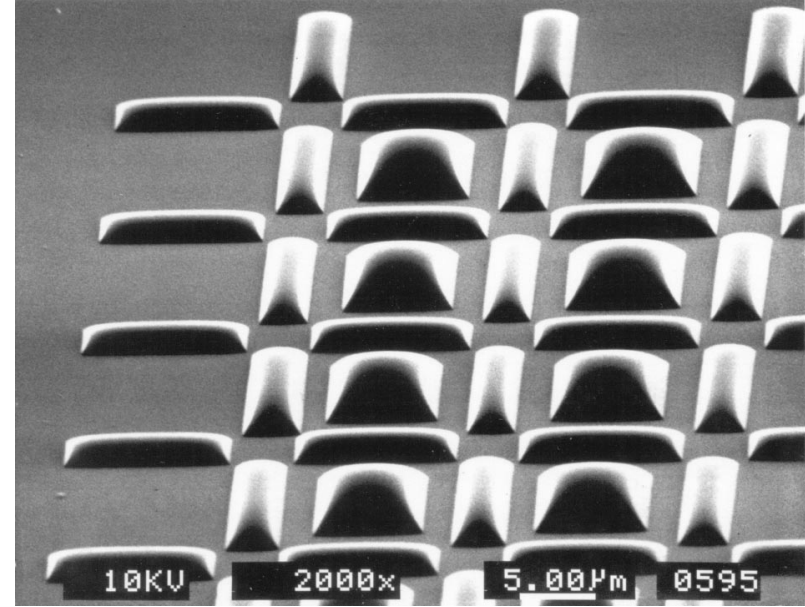

FIG. 3. SEM micrograph of molded $\Delta$ ridges in polycarbonate on a compact disk. The structures have different heights and are incompletely molded, and the aspect ratio is lower than the maximum 0.7 .

ditions. Several hundred disks $80 \mathrm{~mm}$ in diameter (single format) and thickness of $1.2 \mathrm{~mm}$ were produced with cycle times down to $10 \mathrm{~s}$. With careful adjustment of both the mechanical and process parameters, damage to the wafer, e.g., due to wear at the fixation during processing, can be largely avoided, and we were able to increase the number of molded disks per wafer to over 1500 .

\section{CHARACTERIZATION OF MOLDED STRUCTURES}

Molded CDs were analyzed by scanning electron microscopy (SEM) (Topcon ABT60), by atomic force microscopy (Park Scientific Instrument Autoprobe) and by scanning tunneling microscopy (STM) (NanoSurf Easyscan). In order to exclude errors in the measurements because of electrostatic charging of the polymer, the surface was coated with a thin metal film. AFM analysis was carried out using the so-called intermittent contact (IC) or "tapping" mode to avoid possible deformation by tip-ridge interaction. It was found that the precision of the molding by hot embossing could not be matched using the current injection molding process. The bottom of the ridges is well structured, even featuring sub$\mu \mathrm{m}$ details of corners and imperfections in the stamp, but the tips of the ridges are smoothed out (see Fig. 3). The groove fill was found to be strongly dependent on the process parameters and molding materials used. This finding provided a scope for improved replication fidelity, e.g. by changing the mold temperatures and using the height of the molded ridges as a quality parameter of the molding fidelity.

The degree of molding of replicated V-groove structures was compared for different process parameters, sizes and designs. In Fig. 4 the dependence of the structure height on the mold temperature is shown for the DP 1-1265 polycarbonate. From 105 to $130{ }^{\circ} \mathrm{C}$ a significant increase of the molded structure height can be seen. With the current setup and mold temperatures, aspect ratios up to a maximum value of 0.4 were realized. The curve indicates that further im-

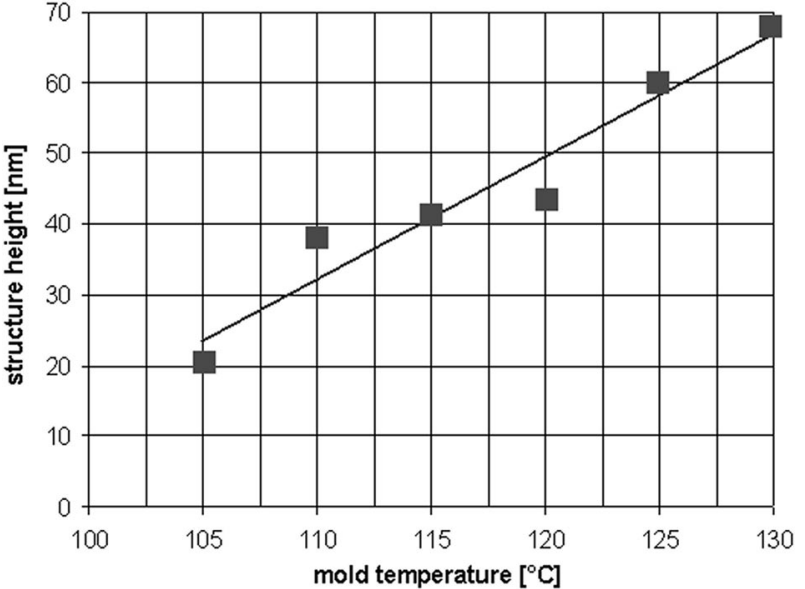

FIG. 4. Structure height of injection molded $\Delta$ ridges dependent on the mold temperature (structure width $200 \mathrm{~nm}$ ).

provements should be achievable by going to higher mold temperatures. The variation of injection speed and dwell pressure has only a minor influence on the molding quality.

A comparison of the molded structures with widths ranging from $120 \mathrm{~nm}$ to $5 \mu \mathrm{m}$ was made. For DP 1-1265 the aspect ratio stays nearly constant for the total range of widths. This indicates that the macroscopic properties of the polymer melt during its viscous flow into a groove rather than the mesoscopic effects are leading to incomplete molding of the structure. As a result, most of the experiments carried out with microstructures represent what happens at all structure sizes. The better molding properties of the DP 1-1265 polycarbonate were exemplified by the constant degree of filling irrespective of distance from the injection point and the orientation of the $\mathrm{V}$ grooves with respect to the direction of the melt flow. ${ }^{12}$ The CD 2005 shows the same fidelity only for $45^{\circ}$ orientation near the injection point and exhibits reduced height for orientations of $10^{\circ}$ and $80^{\circ}$. The structure height was further decreased by $25 \%$ with increasing distance from the injection point $\left(\right.$ at $130{ }^{\circ} \mathrm{C}$ ). These results clearly showed how important the choice of material is for optimum nanomolding.

\section{SHRINKAGE MEASUREMENT}

Thermoplastic polymers suffer from volume contraction when solidifying in closed cavities. A rough estimate of the process shrinkage (the relative lateral size difference between the master and replica) by measuring the outlines of a molded disk gives a value of $2 \%$, which is higher than the $0.6 \%-0.8 \%$ given for polycarbonate in the data sheets. ${ }^{11}$ Our aim was to make a more precise measurement and to determine the local variation of the shrinkage. This is important if identical structures have to be placed at different positions on the $\mathrm{CD}$. In addition to the structured areas, we generated an array of precisely positioned measurement marks over the whole wafer surface. After the injection molding process the absolute position of the molded marks was detected and compared with the positions of the original marks. Our electron beam lithography writer Leica LION LV1 offers opti- 


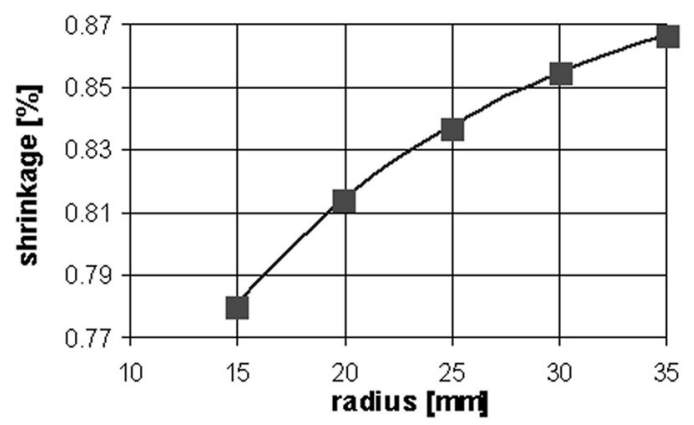

FIG. 5. Radial shrinkage of a compact disk dependent on the distance from the injection point at the disk center.

mal conditions for such measurements, because it can be used in both the writing mode as well as in the SEM mode. Its interferometrically controlled stage with high position accuracy can be used for measurement of local deviation of the marks with sub- $\mu \mathrm{m}$ precision. The detection of the mark edges was facilitated by an automated edge extraction mechanism, which makes it possible to detect the $x, y$ positions of the marks all over the wafer with an average precision of $100 \mathrm{~nm}$. The deviations in the $z$ direction were controlled to guarantee that the height deviations did not affect the measurements in the lateral direction.

The resulting coordinates were analyzed using MATHCAD software. The shrinkage was found to be mainly radial and dependent on the distance from the injection point (see Fig. 5 ). For device areas of a few $\mathrm{mm}^{2}$, only the mean value of the shrinkage at a specific radius of the disk has to be considered and, as demonstrated in Sec. VII, makes it possible to fabricate elements with extremely high precision requirements.

\section{MANUFACTURING OF CALIBRATION STRUCTURES}

Two-dimensional crossed gratings with square profiles are well suited for lateral calibration of the piezo actuators of scanning probe microscopes. Ideally, the dimensions of the features of the calibration samples should be the same order of magnitude as the structural features to be characterized, i.e., ranging from a few nm to several $\mu \mathrm{m}$. For calibration of the scan ranges around $1 \mu \mathrm{m}$ (e.g., required for the low-cost STM Easyscan), a calibration structure with a period of 160 $\mathrm{nm}$ over a structured field size of $2 \times 2 \mathrm{~mm}^{2}$ was designed and manufactured (see Fig. 6) and is now commercially available. ${ }^{13}$ The plastic replicas were investigated by AFM and STM techniques and showed a high fidelity reproduction of the submicron structures of the silicon master. We estimate the accuracy of the STM measurement to be $\pm 5 \mathrm{~nm}$ over a measuring field of $500 \times 500 \mathrm{~nm}^{2}$. High precision control measurements with an interferometric AFM long range profiler with an uncertainty of $0.08 \mathrm{~nm}$ (at $95 \%$ confidence level) of the molded grating resulted in a value of $158.6 \mathrm{~nm}$, which is in excellent agreement with the nominal value of $160 \mathrm{~nm}$ of the master, taking into account shrinkage of the polymer during the molding process. As shown before, the

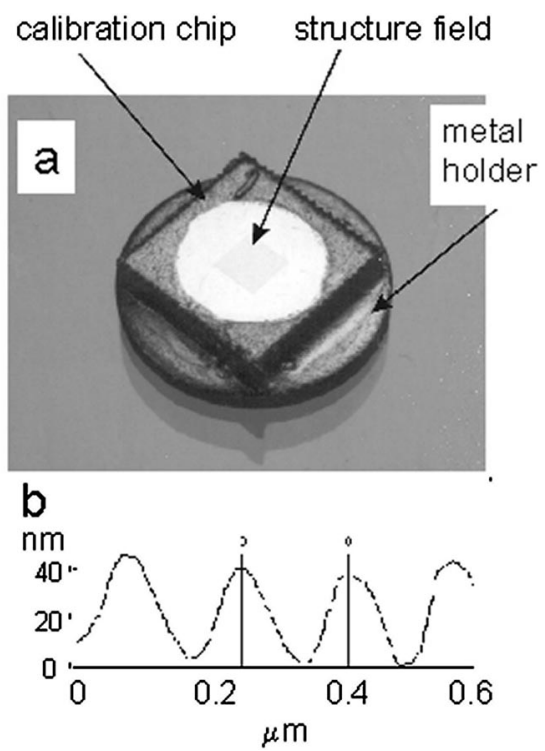

FIG. 6. (a) Photograph of an injection molded calibration structure $(6 \times 6$ $\mathrm{mm}^{2}$ on a metal holder with a structured field size of $2 \times 2 \mathrm{~mm}^{2}$ ). (b) AFM line profile measurement of an array of continuous (adjacent) $\Delta$ ridges on a $\mathrm{CD}$ (period $160 \mathrm{~nm})$.

shrinkage has an average value of $0.85 \%$ for the structure used and, by including this in structure fabrication, the accuracy of a calibration could probably be reduced to a fraction of a nm. The $\Delta$-ridge structures described in Sec. IV can also be used as calibration structures. An AFM measurement of an array of $\Delta$-shaped line structures on a CD (period $160 \mathrm{~nm})$ is shown in Fig. 6.

\section{CONCLUSIONS}

In this work we have shown an initial approach for quantitative measurement of the replication fidelity of injection molded nanostructures. Our main conclusions are as follows.

(1) The dominant factor for the molding of nanostructures is the strong dependence of the melt viscosity on the mold temperature. The molding fidelity increased constantly up to temperatures slightly below the glass transition temperature of the polymer. Further improvements can be expected for higher mold temperatures.

(2) The aspect ratio of the molded structures is nearly constant for different structure sizes on the same molded wafer. We are therefore able to make most of our experiments with microstructured polymer ridges.

(3) The experiments show significantly better molding uniformity of the less viscous Makrolon DP 1-1265, which therefore makes it more suitable for nanomolding than the CD 2005 polycarbonate.

Our experiments showed that masters with $\mathrm{V}$ grooves can be used both for the testing of new materials and improvement of the fidelity of molding in the micro- and nanoranges. The $\mathrm{V}$ grooves were not molded with the same fidelity as the previously molded $25 \mathrm{~nm}$ rectangular structures. ${ }^{6}$ It appears that it is the shape rather than the size of the structure which 
is important here and that we have reached a limit where the viscosity of the polymer is not sufficiently low to fill the entire cavity.

The reason why replication of box shaped structures below $100 \mathrm{~nm}$ is easier to achieve than $\mathrm{V}$ grooves needs to be the subject of further investigations. It would be beneficial to carry out nanorheological experiments, comparing the flow of the polymer into different structure geometries (different angles or aspect ratios) of the mold. Improvement of the molding precision with the current setup is likely, if higher molding temperatures or other processes such as compression molding or a variotherm process are used. Furthermore, materials with significantly better flow properties will lead to improvements in the replication fidelity.

In addition to applications in which either the smooth walls of the ridges (e.g., for reflectors) or the tip (e.g., for substrates with antiwetting properties) can be used, molded $\mathrm{V}$ grooves are also very suitable as calibration structures for SPM. The inclined sidewalls of these structures are very advantageous for easy measurement in comparison to box shaped nanostructures. The more pronounced edges at the bottom of the structures could be used as a precise determination of the period of a structure. Precise measurement of the local shrinkage of the disk means that we are able to fabricate many identical gratings with precisions in the sub-nm range in a mass fabrication process.

\section{ACKNOWLEDGMENTS}

The following people are gratefully acknowledged for their help and valuable contributions: D. Bächle, F. Glaus, B.
Haas, P. Häberling, L. J. Heyderman, R. W. Jaszewski, B. Ketterer, and T. Mezzacasa, PSI; O. Häfeli, F. H. Aargau, J. Krummenacher, O. Fischer and M. Wey, AWM Werkzeugbau AG; S. Köppel, N. Kudlik, and W. Zangerle, Netstal Maschinen AG; L. Scandella, Nanosurf AG, and F. Meli, Swiss Federal Office of Metrology (OFMET). Part of this work was supported by the Swiss National Science Foundation within the framework NFP 36 Nanosciences.

${ }^{1}$ L. Reimer and C. Schulte, Naturwissenschaften 53, 489 (1996).

${ }^{2}$ P. R. Krauss and S. Y. Chou, Appl. Phys. Lett. 71, 3174 (1997).

${ }^{3}$ B. W. Chui, T. D. Stowe, S. J. Yongho, K. E. Goodson, T. W. Kenny, H. J. Mamin, B. D. Terris, R. P. Ried, and D. Rugar, J. Microelectromech. Syst. 7, 69 (1998).

${ }^{4}$ R. W. Jaszewski, H. Schift, J. Gobrecht, and P. Smith, Microelectron. Eng. 41/42, 575 (1998).

${ }^{5}$ D. MacIntyre and S. Thomas, Microelectron. Eng. 41/42, 211 (1998).

${ }^{6}$ H. Schift, C. David, M. Gabriel, J. Gobrecht, L. J. Heyderman, W. Kaiser, S. Köppel, and L. Scandella, Microelectron. Eng. 53, 171 (2000).

${ }^{7}$ L. J. Heyderman, H. Schift, C. David, J. Gobrecht, and T. Schweizer, Microelectronic Eng. (to be published).

${ }^{8}$ F. Nikolajeff, S. Jacobsson, S. Hard, A. Billman, L. Lundbladh, and C. Lindell, Appl. Opt. 36, 4655 (1997).

${ }^{9}$ Mikromechanik, edited by A. Heuberger (Springer, Berlin, 1989).

${ }^{10} \mathrm{H}$. Schift, R. W. Jaszewski, C. David, and J. Gobrecht, Microelectron. Eng. 46, 121 (1999).

${ }^{11}$ Bayer AG, Polymer Division, Application Notes Nos. ATI $8016 \mathrm{~d}$ and e (1995).

${ }^{12}$ A. D'Amore, D. Simoneta, W. Kaiser, H. Schift, and M. Gabriel, Kunststoffe (to be published).

${ }^{13}$ J. Gobrecht, H. Schift, C. David, W. Kaiser, A. D'Amore, D. Simoneta, and L. Scandella, PTB-Berichte PTB-F-39, 1 (2000). 